\title{
Studies on Genetic Variability, Correlation and Path Analysis in Lentil (Lens culinaris Medik.) Genotypes
}

\author{
Jitendar Kumar Meena ${ }^{1 *}$, Khajan Singh ${ }^{1}$, P.K.P. Meena ${ }^{1}$, \\ Rajesh Kumar ${ }^{2}$ and Deepak Meena ${ }^{1}$
}

${ }^{1}$ Department of Genetics and Plant Breeding, ${ }^{2}$ Department of Agronomy, College of Agriculture Ummedganj, Kota, Agriculture University Kota, India

*Corresponding author

\section{Ke y w o r d s \\ Genetic variability, Correlation and path analysis, Lentil}

Article Info

Accepted:

17 August 2020

Available Online:

10 September 2020

\section{A B S T R A C T}

\begin{abstract}
Present study was conducted on 155 lentil genotypes including five check varieties viz., Kota Masoor-1, Kota Masoor-2, JL-3, IPL-316 and L-4076 for genetic evaluation at experimental field of AICRP on MULLaRP, Agricultural Research Station, Ummedganj, Agriculture University, Kota, Rajasthan, India. These genotypes were sown in Augmented Randomized Complete Block Design during Rabi, 2019-20. Observations were recorded for thirteen different yield and its related parameters. The analysis of variance indicated the existence of significant differences among genotypes for all the characters studied. Biological yield per plant, number of peduncles per plant, number of pods per plant, number of primary branches per plant, number of secondary branches per plant and seed yield per plant recorded high GCV, high heritability and high genetic gain. The correlation coefficient analysis indicated that biological yield per plant, harvest index, number of peduncles per plant, number of pods per plant, number of primary branches per plant and plant height exhibited significant correlation with seed yield per plant. Path analysis exhibited that out of seven characters which were positively correlated with seed yield per plant, only three characters viz., biological yield per plant, harvest index and number of pods per plant had positive and high direct effect. The positive correlation of other characters was mainly due to their indirect effects via biological yield per plant, harvest index and number of pods per plant. Hence these characters may be considered for further breeding programme to improve the seed yield in lentil.
\end{abstract}

\section{Introduction}

Lentil (Lens culinaris Medik.) is an important pulse crop and plays an important role in human, animal feeding and soil improvement. It is cultivated in the semi- arid regions of the world particularly in the Indian Sub-continent and the dry areas of Middle East (Malik, 2005). It is a short stature; annual, selfpollinate high value crop which has great significance in cereal based cropping system. It belongs to Family Fabaceae sub family Papilionaceae. Lentil is bushy, autogamous diploid crop $(2 n=2 x=14)$. The total area under 
lentil in India was 14.94 lakh hectares with a total production of 15.06 lakh tones with productivity of $1008 \mathrm{~kg} /$ hectare, during 201718 (Anonymous, 2019) and contributes about $7.88 \%$ in total pulse production. Madhya Pradesh, Uttar Pradesh, Bihar and West Bengal are major lentil producing states. Lentil thrives well in sub marginal lands with low inputs under water limited conditions. Rajasthan has large area of undulated, uncultivable waste land, sub-marginal soils with low moisture content. Under such situations lentil as a pulse crop can be well fitted in cropping system providing nutritional security to resources poor farmers in Rajasthan. It is grown in Bundi, Kota, Pratapgarh, Bhilwara, Jhalawar and Bharatpur districts of Rajasthan covering the total area of 0.31 lakh hectare, producing 0.43 lakh tonnes with productivity of $1408 \mathrm{~kg} /$ hectare (Anonymous, 2019).

Genetic variability is prerequisite for its possible utilization in tailoring high yielding genotypes. Knowledge about the magnitude of genetic variability and heritability of characters is essential for a successful breeding programme. Information about the correlation between yield and other characters greatly help the breeder in selecting useful characters for enhancing yield. The study of genetic variability with the help of suitable genetic parameters such as coefficient of variation, heritability estimates and genetic advance and correlation coefficient is useful for genetic up-gradation of yield in lentil.

\section{Materials and Methods}

The present research study was carried out during Rabi, 2019-20 at experimental field of AICRP on MULLaRP, Agricultural Research Station, Ummedganj, Agriculture University, Kota, Rajasthan, India. The experiment was laid out in an Augmented Randomized Complete Block Design (Federer, 1956). The material was sown in 10 blocks. Each block had 4 meter long 20 lines placed $30 \mathrm{~cm}$. apart. Thus 15 genotypes and 5 checks were sown in each block. The checks were common in the blocks and they were randomized among themselves. Observation were recorded on thirteen characters viz., days to 50 per cent flowering, days to maturity, plant height, number of primary branches per plant, number of secondary branches per plant, number of peduncles per plant, number of pods per plant, number of seeds per pod, 100seed weight, biological yield per plant, harvest index, protein content and seed yield per plant. Mean data were recorded on five randomly selected plants except days to 50 per cent flowering and days to maturity in which observation were recorded on population basis. Analysis of variance for the design of experiment was done using the method suggested by Federer (1956). The genotypic and phenotypic correlation coefficients were calculated using the formula given by Johnson et al., (1955). The estimates of direct and indirect effects were calculated by the path coefficient analysis as suggested by Wright (1921) and elaborated by Dewey and $\mathrm{Lu}$ (1959).

\section{Results and Discussion}

Estimates of genotypic and phenotypic variances indicated that in general the phenotypic variances were slightly higher than the corresponding genotypic variance indicating the role of environmental factors on the character expression. High magnitude of GCV (more than 20\%) was recorded for seed yield per plant, biological yield per plant, 100 -seed weight, number of pods per plant, number of peduncles per plant, number of secondary branches per plant and number of primary branches per plant indicating a good deal of variability in these characters. Such results were also reported by Sirohi et al., (2007), Tyagi and Khan (2010), Singh et al., (2012). 
The estimates of heritability were higher $(>80$ per cent) for number of peduncles per plant, number of pods per plant, number of secondary branches per plant, number of primary branches per plant and biological yield per plant indicating that these characters were less affected by environment and direct selection for these yield contributing traits would be effective for future improvement in yield. Such results were also reported by Chakraborty and Haque (2000), Rasheed et al., (2008).

Seed yield per plant revealed significant and positive correlation with biological yield per plant, number of pods per plant, number of peduncles per plant, number of primary branches per plant, harvest index and plant height. Mutual correlations among most of these traits were also positive except with harvest index. This suggests that simultaneous selection for these traits will have a better efficiency for improving the seed yield per plant. Similar results were reported by Chakraborty and Haque (2000), Rasheed et al., (2008), Deb et al., (2009), Latif et al., (2010) and Tyagi and Khan (2010). Number of secondary branches per plant did not show any significant correlation with seed yield per plant but it exhibited significant correlation with number of peduncles per plant, number of pods per plant and number seeds per pod, which were significantly correlated with seed yield per plant. This indicates that a greater number of primary branches per plant and number of secondary branches per plant were associated with greater number of peduncles per plant and number of pods per plant and intern more seed yield per plant. Similar results were also reported by Rasheed et al., (2008), Tadesse et al., (2014) and Sakthivel et $a l$. , (2019). The hundred-seed weight also did not show significant correlation with seed yield per plant but it was significantly correlated with harvest index, which was significantly correlated with seed yield per plant, hence it contributes to the seed yield per plant.

Days to $50 \%$ flowering did not show any correlation with seed yield per plant. Through it showed significant and positive correlation with days to maturity at both levels and negatively correlated with harvest index which was positively correlated with seed yield per plant. Thus days to $50 \%$ flowering did not exhibit any significance towards seed yield per plant. Similar results were reported by Younis et al., (2008), Sharma et al., (2014), Tabti et al., (2018) and Chowdhury et al., (2019).

The number of secondary branches per plant did not exhibit any correlation with seed yield per plant, though it had positive correlation with number of seeds per pod, number of peduncles per plant and number of pods per plant at both genotypic and phenotypic levels, which were positively correlated with seed yield per plant. Similar trends of the association between number of pods per plant and number of seeds per pod were also reported by Pandey et al., (2017) and Hussan et al., (2018)

100-seed weight did not exhibit any correlation with seed yield per plant, though it was positively correlated with harvest index at genotypic level, which was positively correlated with seed yield. It had negative correlation with number of primary branches per plant, number of peduncles per plant and number of pods per plant. These results were similar with Rasheed et al., (2008) and Younis et al., (2008).

Protein content showed positive correlation with seed yield and other component characters thought it was non-significant, hence high proteinous genotype may be used with high seed yield genotypes in further breeding programme (Table 1-4). 
Table.1 General mean, range, variance, genotypic and phenotypic coefficients of variation, heritability (broad sense) and genetic advance as percentage of mean for different characters in lentil

\begin{tabular}{|c|c|c|c|c|c|c|c|c|}
\hline \multirow[t]{2}{*}{ S. No } & \multirow{2}{*}{ Characters } & \multirow[t]{2}{*}{ Mean } & \multicolumn{2}{|c|}{ Range } & \multirow{2}{*}{$\begin{array}{l}\text { Genotypic } \\
\text { coefficient of } \\
\text { variation } \\
(\%)\end{array}$} & \multirow{2}{*}{$\begin{array}{l}\text { Phenotypic } \\
\text { coefficient of } \\
\text { variation } \\
(\%)\end{array}$} & \multirow{2}{*}{$\begin{array}{c}\text { Heritability } \\
\text { in broad } \\
\text { sense } \\
(\%)\end{array}$} & \multirow{2}{*}{$\begin{array}{c}\text { Genetic } \\
\text { advance as } \\
\text { per cent of } \\
\text { mean }\end{array}$} \\
\hline & & & Min. & Max. & & & & \\
\hline 1. & $\begin{array}{l}\text { Days to } 50 \% \\
\text { flowering }\end{array}$ & 60.21 & 53.98 & 72.18 & 5.84 & 7.54 & 59.03 & 9.15 \\
\hline 2. & Days to maturity & 106.46 & 96.14 & 130.34 & 4.73 & 6.40 & 54.66 & 7.20 \\
\hline 3. & Plant height (cm) & 37.17 & 26.56 & 45.16 & 9.60 & 11.66 & 67.76 & 16.26 \\
\hline 4. & $\begin{array}{l}\text { Number of primary } \\
\text { branches per plant }\end{array}$ & 10.03 & 4.87 & 18.07 & 31.86 & 35.20 & 81.94 & 59.21 \\
\hline 5. & $\begin{array}{l}\text { Number of secondary } \\
\text { branches per plant }\end{array}$ & 10.23 & 3.11 & 18.51 & 33.59 & 36.62 & 84.13 & 59.03 \\
\hline 6. & $\begin{array}{l}\text { Number of peduncles } \\
\text { per plant }\end{array}$ & 29.85 & 12.07 & 69.43 & 31.97 & 33.90 & 88.92 & 62.26 \\
\hline 7. & $\begin{array}{l}\text { Number of pods per } \\
\text { plant }\end{array}$ & 47.05 & 17.50 & 122.88 & 36.25 & 39.22 & 85.45 & 68.89 \\
\hline 8. & $\begin{array}{l}\text { Number of seeds per } \\
\text { pod }\end{array}$ & 1.37 & 1.13 & 1.61 & 7.34 & 8.83 & 69.02 & 12.55 \\
\hline 9. & 100-seed weight (g) & 3.21 & 1.49 & 6.46 & 20.55 & 25.10 & 67.01 & 34.62 \\
\hline 10. & $\begin{array}{l}\text { Biological yield per } \\
\text { plant(g) }\end{array}$ & 4.73 & 1.08 & 10.56 & 35.19 & 38.93 & 81.74 & 65.30 \\
\hline 11. & Harvest index (\%) & 38.92 & 11.81 & 59.77 & 18.92 & 21.43 & 77.93 & 34.41 \\
\hline 12. & Protein content $(\%)$ & 20.85 & 16.24 & 28.88 & 5.58 & 7.31 & 58.25 & 8.78 \\
\hline 13. & $\begin{array}{l}\text { Seed yield per plant } \\
\text { (g) }\end{array}$ & 1.81 & 0.25 & 5.63 & 37.76 & 42.26 & 79.81 & 69.20 \\
\hline
\end{tabular}


Table. 2 Correlation coefficients between different characters in lentil at genotypic level

\begin{tabular}{|c|c|c|c|c|c|c|c|c|c|c|c|c|c|}
\hline Characters & $\begin{array}{c}\text { Days to } \\
50 \% \\
\text { flowering }\end{array}$ & $\begin{array}{l}\text { Days to } \\
\text { maturity }\end{array}$ & $\begin{array}{c}\text { Plant } \\
\text { height }(\mathrm{cm})\end{array}$ & $\begin{array}{l}\text { Number of } \\
\text { primary } \\
\text { branches } \\
\text { per plant }\end{array}$ & $\begin{array}{c}\text { Number of } \\
\text { secondary } \\
\text { branches } \\
\text { per plant }\end{array}$ & $\begin{array}{l}\text { Number of } \\
\text { peduncles } \\
\text { per plant }\end{array}$ & $\begin{array}{c}\text { Number of } \\
\text { pods per } \\
\text { plant }\end{array}$ & $\begin{array}{l}\text { Number of } \\
\text { seeds } \\
\text { per pod }\end{array}$ & $\begin{array}{l}100 \text {-seed } \\
\text { weight }(\mathrm{g})\end{array}$ & $\begin{array}{l}\text { Biological } \\
\text { yield } \\
\text { per } \\
\text { plant }(g)\end{array}$ & $\begin{array}{c}\text { Harvest } \\
\text { index }(\%)\end{array}$ & $\begin{array}{c}\text { Protein } \\
\text { content } \\
(\%)\end{array}$ & $\begin{array}{l}\text { Seed yield } \\
\text { per plant } \\
\text { (g) }\end{array}$ \\
\hline $\begin{array}{l}\text { Days to } 50 \% \\
\text { flowering }\end{array}$ & 1.000 & $0.626 * *$ & -0.012 & -0.153 & 0.025 & 0.066 & 0.008 & 0.029 & -0.063 & 0.032 & $-0.264 * *$ & -0.039 & -0.099 \\
\hline Days to maturity & & 1.000 & -0.002 & 0.099 & -0.039 & 0.072 & 0.080 & -0.067 & -0.081 & 0.033 & -0.104 & 0.091 & -0.029 \\
\hline Plant height (cm) & & & 1.000 & $0.247 * *$ & $0.305 * *$ & $0.246 * *$ & $0.228 * *$ & $0.255^{* *}$ & 0.058 & $0.239 * *$ & 0.010 & -0.065 & $0.238 * *$ \\
\hline $\begin{array}{l}\text { Number of } \\
\text { primary branches } \\
\text { per plant }\end{array}$ & & & & 1.000 & $0.374 * *$ & $0.598 * *$ & $0.661 * *$ & $0.294 * *$ & $-0.250 * *$ & $0.431 * *$ & 0.099 & -0.033 & $0.467 * *$ \\
\hline $\begin{array}{l}\text { Number of } \\
\text { secondary } \\
\text { branches per plant }\end{array}$ & & & & & 1.000 & $0.292 * *$ & $0.211 * *$ & $0.481 * *$ & -0.053 & 0.133 & -0.147 & -0.056 & 0.050 \\
\hline $\begin{array}{l}\text { Number of } \\
\text { peduncles per } \\
\text { plant }\end{array}$ & & & & & & 1.000 & $0.907 * *$ & $0.359 * *$ & $-0.278 * *$ & $0.658 * *$ & 0.075 & 0.080 & $0.689 * *$ \\
\hline $\begin{array}{l}\text { Number of pods } \\
\text { per plant }\end{array}$ & & & & & & & 1.000 & $0.300 * *$ & $-0.348 * *$ & $0.657 * *$ & 0.139 & 0.066 & $0.727 * *$ \\
\hline $\begin{array}{l}\text { Number of seeds } \\
\text { per pod }\end{array}$ & & & & & & & & 1.000 & -0.136 & $0.230 * *$ & -0.123 & 0.011 & $0.185 *$ \\
\hline 100-seed weight $(\mathrm{g})$ & & & & & & & & & 1.000 & -0.002 & $0.171 *$ & 0.003 & 0.076 \\
\hline $\begin{array}{l}\text { Biological yield } \\
\text { per plant }(\mathrm{g})\end{array}$ & & & & & & & & & & 1.000 & $-0.213 * *$ & -0.065 & $0.821 * *$ \\
\hline Harvest index (\%) & & & & & & & & & & & 1.000 & 0.130 & $0.341 * *$ \\
\hline $\begin{array}{l}\text { Protein content } \\
(\%)\end{array}$ & & & & & & & & & & & & 1.000 & 0.021 \\
\hline $\begin{array}{l}\text { Seed yield per } \\
\text { plant }(\mathrm{g})\end{array}$ & & & & & & & & & & & & & 1.000 \\
\hline
\end{tabular}


Table.3 Correlation coefficients between different characters in lentil at phenotypic level

\begin{tabular}{|c|c|c|c|c|c|c|c|c|c|c|c|c|c|}
\hline Characters & $\begin{array}{c}\text { Days to } \\
50 \% \\
\text { flowering }\end{array}$ & $\begin{array}{l}\text { Days to } \\
\text { maturity }\end{array}$ & $\begin{array}{c}\text { Plant } \\
\text { height } \\
\text { (cm) }\end{array}$ & $\begin{array}{c}\text { Number } \\
\text { of } \\
\text { primary } \\
\text { branches } \\
\text { per plant }\end{array}$ & $\begin{array}{r}\text { Number of } \\
\text { secondary } \\
\text { branches } \\
\text { per plant }\end{array}$ & $\begin{array}{r}\text { Number of } \\
\text { peduncles } \\
\text { per plant }\end{array}$ & $\begin{array}{c}\text { Number } \\
\text { of pods } \\
\text { per plant }\end{array}$ & $\begin{array}{r}\text { Number } \\
\text { of seeds } \\
\text { per pod }\end{array}$ & $\begin{array}{c}\text { 100-seed } \\
\text { weight } \\
(\mathrm{g})\end{array}$ & $\begin{array}{l}\text { Biological } \\
\text { yield } \\
\text { per } \\
\text { plant }(g)\end{array}$ & $\begin{array}{c}\text { Harvest } \\
\text { index } \\
(\%)\end{array}$ & $\begin{array}{c}\text { Protein } \\
\text { content } \\
(\%)\end{array}$ & $\begin{array}{l}\text { Seed } \\
\text { yield per } \\
\text { plant } \\
(\mathrm{g})\end{array}$ \\
\hline $\begin{array}{l}\text { Days to } 50 \% \\
\text { flowering }\end{array}$ & 1.000 & $0.661 * *$ & -0.021 & -0.131 & -0.008 & 0.106 & 0.039 & 0.001 & -0.092 & 0.074 & $0.251 * *$ & -0.022 & -0.050 \\
\hline Days to maturity & & 1.000 & -0.107 & -0.075 & -0.126 & 0.103 & 0.090 & -0.127 & -0.081 & 0.083 & -0.104 & 0.036 & 0.026 \\
\hline Plant height (cm) & & & 1.000 & $0.401 * *$ & $0.381 * *$ & 0.093 & 0.114 & $0.417 * *$ & 0.084 & 0.144 & 0.055 & -0.037 & $0.173 *$ \\
\hline $\begin{array}{l}\text { Number of } \\
\text { primary branches } \\
\text { per plant }\end{array}$ & & & & 1.000 & $0.521 * *$ & $0.408 * *$ & $0.493 * *$ & $0.470 * *$ & $0.247 * *$ & $0.338 * *$ & 0.082 & 0.002 & $0.368 * *$ \\
\hline $\begin{array}{l}\text { Number of } \\
\text { secondary } \\
\text { branches per plant }\end{array}$ & & & & & 1.000 & $0.162 *$ & 0.119 & $0.615 * *$ & -0.055 & 0.106 & -0.140 & 0.047 & 0.029 \\
\hline $\begin{array}{l}\text { Number of } \\
\text { peduncles per } \\
\text { plant }\end{array}$ & & & & & & 1.000 & $0.910 * *$ & $0.198 *$ & $0.248 * *$ & $0.673 * *$ & 0.116 & 0.101 & $0.727 * *$ \\
\hline $\begin{array}{l}\text { Number of pods } \\
\text { per plant }\end{array}$ & & & & & & & 1.000 & 0.150 & $0 . \overline{338 * *}$ & $0.680 * *$ & $0.189 *$ & 0.081 & $0.775 * *$ \\
\hline $\begin{array}{l}\text { Number of seeds } \\
\text { per pod }\end{array}$ & & & & & & & & 1.000 & -0.137 & $0.165^{*}$ & -0.090 & 0.048 & 0.139 \\
\hline 100-seed weight $(\mathrm{g})$ & & & & & & & & & 1.000 & 0.045 & 0.112 & -0.031 & 0.078 \\
\hline $\begin{array}{l}\text { Biological yield per } \\
\operatorname{plant}(\mathrm{g})\end{array}$ & & & & & & & & & & 1.000 & $-0.196^{*}$ & -0.074 & $0.834 * *$ \\
\hline Harvest index (\%) & & & & & & & & & & & 1.000 & 0.080 & $0.334 * *$ \\
\hline $\begin{array}{l}\text { Protein content } \\
(\%)\end{array}$ & & & & & & & & & & & & 1.000 & -0.012 \\
\hline $\begin{array}{l}\text { Seed yield per } \\
\text { plant }(g)\end{array}$ & & & & & & & & & & & & & 1.000 \\
\hline
\end{tabular}

$*$, ** significant at $5 \%$ and $1 \%$ levels, respectively 
Table.4 Genotypic path coefficient analysis showing direct and indirect effects of different characters on seed yield in lentil (Lens culinaris Medik.)

\begin{tabular}{|c|c|c|c|c|c|c|c|c|c|c|c|c|c|}
\hline Characters & $\begin{array}{c}\text { Days to } \\
50 \% \\
\text { flowering }\end{array}$ & $\begin{array}{l}\text { Days to } \\
\text { maturity }\end{array}$ & $\begin{array}{c}\text { Plant } \\
\text { height } \\
(\mathrm{cm})\end{array}$ & $\begin{array}{c}\text { Number } \\
\text { of } \\
\text { primary } \\
\text { branches } \\
\text { per plant }\end{array}$ & $\begin{array}{l}\text { Number of } \\
\text { secondary } \\
\text { branches } \\
\text { per plant }\end{array}$ & $\begin{array}{c}\text { Number } \\
\text { of } \\
\text { peduncle } \\
\text { s per } \\
\text { plant }\end{array}$ & $\begin{array}{c}\text { Number } \\
\text { of pods } \\
\text { per plant }\end{array}$ & $\begin{array}{l}\text { Number } \\
\text { of seeds } \\
\text { per pod }\end{array}$ & $\begin{array}{l}\text { 100-seed } \\
\text { weight } \\
\text { (g) }\end{array}$ & $\begin{array}{l}\text { Biologica } \\
\text { l yield } \\
\text { per } \\
\text { plant }(g)\end{array}$ & $\begin{array}{c}\text { Harvest } \\
\text { index } \\
(\%)\end{array}$ & $\begin{array}{c}\text { Protein } \\
\text { content } \\
(\%)\end{array}$ & $\begin{array}{c}\text { Correlati } \\
\text { on } \\
\text { With } \\
\text { seed } \\
\text { Yield per } \\
\text { Plant (g) }\end{array}$ \\
\hline $\begin{array}{l}\text { Days } \\
\text { to50\% flowering }\end{array}$ & 0.0206 & -0.0133 & -0.0001 & 0.0021 & -0.0006 & -0.0004 & 0.0009 & 0.0010 & -0.0015 & 0.0280 & -0.1351 & -0.0002 & -0.099 \\
\hline Days to maturity & 0.0129 & -0.0213 & 0.0001 & -0.0014 & 0.0009 & -0.0005 & 0.0084 & -0.0024 & -0.0019 & 0.0290 & -0.0533 & 0.0004 & -0.029 \\
\hline Plant height (cm) & -0.0002 & 0.0001 & 0.0052 & -0.0034 & -0.0072 & -0.0016 & 0.0238 & 0.0090 & 0.0014 & 0.2067 & 0.0050 & -0.0003 & $0.238 * *$ \\
\hline $\begin{array}{l}\text { Number of primary } \\
\text { branches per plant }\end{array}$ & -0.0031 & -0.0021 & 0.0013 & -0.0137 & -0.0088 & -0.0040 & 0.0692 & 0.0104 & -0.0059 & 0.3733 & 0.0505 & -0.0002 & $0.467 * *$ \\
\hline $\begin{array}{l}\text { Number of } \\
\text { secondary branches } \\
\text { per plant }\end{array}$ & 0.0005 & 0.0008 & 0.0016 & -0.0051 & -0.0235 & -0.0019 & 0.0221 & 0.0170 & -0.0013 & 0.1149 & -0.0752 & -0.0003 & 0.050 \\
\hline $\begin{array}{l}\text { Number of } \\
\text { peduncles per plant }\end{array}$ & 0.0014 & -0.0015 & 0.0013 & -0.0082 & -0.0069 & -0.0066 & 0.0949 & 0.0127 & -0.0066 & 0.5698 & 0.0386 & 0.0004 & $0.689 * *$ \\
\hline $\begin{array}{l}\text { Number of pods per } \\
\text { plant }\end{array}$ & 0.0002 & -0.0017 & 0.0012 & -0.0090 & -0.0050 & -0.0060 & 0.1047 & 0.0106 & -0.0082 & 0.5690 & 0.0711 & 0.0003 & $0.727 * *$ \\
\hline $\begin{array}{l}\text { Number of seeds } \\
\text { per pod }\end{array}$ & 0.0006 & 0.0014 & 0.0013 & -0.0040 & -0.0113 & -0.0024 & 0.0314 & 0.0353 & -0.0032 & 0.1987 & -0.0630 & 0.0001 & $0.185^{*}$ \\
\hline 100-seed weight (g) & -0.0013 & 0.0017 & 0.0003 & 0.0034 & 0.0013 & 0.0018 & -0.0364 & -0.0048 & 0.0237 & -0.0016 & 0.0874 & 0.0001 & 0.076 \\
\hline $\begin{array}{l}\text { Biological yield per } \\
\text { plant(g) }\end{array}$ & 0.0007 & -0.0007 & 0.0013 & -0.0059 & -0.0031 & -0.0044 & 0.0688 & 0.0081 & 0.0001 & 0.8656 & -0.1091 & -0.0003 & $0.821 * *$ \\
\hline Harvest index (\%) & -0.0054 & 0.0022 & 0.0001 & -0.0014 & 0.0035 & -0.0005 & 0.0145 & -0.0043 & 0.0040 & -0.1844 & 0.5124 & 0.0006 & $0.341 * *$ \\
\hline Protein content $(\%)$ & -0.0008 & -0.0019 & -0.0003 & 0.0005 & 0.0013 & -0.0005 & 0.0070 & 0.0004 & 0.0001 & -0.0559 & 0.0664 & 0.0048 & 0.021 \\
\hline
\end{tabular}


These results were similar with Hussan et al., (2018). Path analysis was computed at genotypic level only by taking seed yield per plant as dependent variable to partition the correlation coefficient into the measures of direct and indirect effects in order to determine the contribution of different characters towards seed yield. Out of the seven characters viz., plant height, number of primary branches per plant, number of peduncles per plant, number of pods per plant, number of seeds per pod, biological yield per plant and harvest index, which were positively correlated with seed yield per plant only three characters viz., biological yield per plant, harvest index and number of pods per plant had positive and high direct effect. Biological yield per plant and number of pods per plant also exerted indirect effect through each other. The positive correlation of other traits was mainly due to their indirect effects via, biological yield per plant, number of pods per plant and harvest index. Biological yield per plant and harvest index exhibited negative indirect effect with each other. Higher direct effect of biological yield per plant was also reported by Younis et al., (2008), Sharma et al., (2014), Dalbeer et al., (2015), Pandey et al., (2017) and Sakthivel et al., (2019.

Plant height showed significant positive correlation with seed yield per plant, although its direct effect was less. Plant height contributed seed yield due to indirect effect via., biological yield per plant and number of pods per plant, Similar findings were confirmed by Bicer and Sakar (2008), Sharma et al., (2014) and Dalbeer et al., (2015).

Number of primary branches per plant, number of peduncles per plant and number of secondary branches per plant exhibited negative direct effect on seed yield per plant. But positive indirect effect on seed yield was observed through biological yield per plant, number of pods per plant, harvest index and number of seeds per pod. Similar finding was reported by Dalbeer et al., (2015), Pandey et al., (2017) and Tabti et al., (2018).

The number of seeds per pod had positive direct effect and indirect effects via., biological yield per plant and number of pods per plant. The indirect effects of number of seeds per pod through harvest index and number of secondary branches per plant were negative. Similar finding was reported by Dalbeer et al., (2015) and Tabti et al., (2018).

In this study the genotypic residual effect ( $\mathrm{R}$ $=0.0423)$ was found, indicated that about 95 per cent variability of seed yield could be attributed to the characters under study and only a small fraction i.e. 5 per cent variability of seed yield may be contributed by some other characters. The residual component of path analysis exhibited that about 95 per cent variability of seed yield per plant was accounted by these twelve studied characters.

Thus, the path analysis for seed yield per plant exhibited that the seed yield was mainly a product of direct as well as indirect effects of biological yield per plant, harvest index and number of pods per plant. Therefore, the variability for biological yield per plant, harvest index and number of pods per plant should be exploited for prospect of improvement for seed yield.

\section{References}

Anonymous. (2019). Pulses revolution-from food to nutritional security. Ministry of Agriculture and Farmers Welfare, (Dept. of Agriculture, Corporation \& Farmers Welfare), Govt. of India. 20pp.

Arumuganathan, K. and Earle, E. D. (1991). Nuclear DNA content of some important plant species. Plant Molecular Biology, 9: 208-218.

Bicer, B. T. and Sakar, D. (2008). Heritability 
and path analysis of some economical characteristics in lentil. Journal of Central European Agriculture, 9(1): 175-179.

Chakraborty, M. and Haque, M. F. (2000). Genetic variability and component analysis in lentil (Lens culinaris Medik.). Journal of Research, Birsa Agricultural University, 12(2):199-204.

Chowdhury, M. M., Haque, M. A., Malek, M. A., Rasel, M. and Ahamed, K.U. (2019). Genetic variability, correlation and path coefficient analysis for yield and yield components of selected lentil (Lens culinaris M.) genotypes. Fundamental and Applied Agriculture, 4(2): 769-776.

Dalbeer, Nath, S., Verma, O. P., Kavita, and Kumar, K. (2015). Correlation and path coefficient analysis for yield attributes in lentil. International Journal of Science and Research,4(8): 158-160.

Deb, A. C., Dutta, A. K. and Khaleque, M. A. (2009). Correlation and Path coefficient analysis in lentil (Lens culinaris Medik.). Journal Sher-e-Bangla Agriculture University, 3(2): 24-29.

Dewey, D. R. and Lu, K. H. (1959). A correlation and path coefficient analysis of components of crested wheat grass seed production. Journal of Agronomy, 51: 515-518.

Federer, W.T. (1956). Augmented designs. Hawain planters record, Inc., New York, 20: 191-207.

Hussan, S. U., Khuroo, N. S., Lone, A. A., Dar, Z. A., Dar, S. A. and Dar, M. S. (2018). Study of variability and association analysis for various agro morphological traits in lentil (Lens $\begin{array}{lll}\text { culinaris } & \text { M.). Journal of }\end{array}$ Pharmacognosy and Phytochemistry, 7(2): 2172-2175.

Johnson, H.W., Robinson, H. F. and Comstock, R.E. (1955). Estimates of genetic and environmental variability in soybean. Agronomy Journal, 47: 314318.

Latif, M. A., Hassan, M. M. and Sultana, N. (2010). Genetic variability, character association and path coefficient analysis in lentil (Lens culinaris Medik). Bangladesh Journal Environmental Science, 18: 49-51.

Malik, R. (2005). Genetic Divergence Analysis in Lentil (Lens culinaris Medik.). M.Sc. Department of Agricultural Botany. Chaudhary Charan Singh University, Meerut (U.P.), India, pp.1.

Muehlbauer, F. J. (1991). Use of introduced germplasm in cool season food legume cultivar development. In H. L. Shands \& L. E. Wiesner (Eds.), Use of Plant Introductions in Cultivar Development (Part 2). Crop Sci. Soc. Amer. Social publication, 20, 49-73.

Pandey, S., Kureshi, S. P., and Bhatore, A. (2017). Studies on genetic variability and interrelationship among the different traits in exotic lines of lentil (Lens culinaris Medik). Plant Archives, 17(2): 1164-1170.

Rasheed, S., Hanif, M., Sadiq, S., Abbas, G., Asghar, M. J. and Haq, M. A. (2008). inheritance of seed yield and related traits in some lentil (Lens culinaris Medik.) genotypes. Pakistan Journal of Agriculture Science, 45(3): 49-52.

Sakthivel, G., Jeberson, S., Singh, N. B., Sharma, P. R., Kumar, S., Jalaj, V. K., Sinha, B. and Singh N. O. (2019). Genetic variability, correlation and path analysis in lentil germplasm (Lens culinaris Medik.). The Pharma Innovation Journal, 8(6): 417-420.

Sharma, V., Paswan, S. K., Singh, V. K. and Khandagale, S. (2014). Correlation and path coefficient analysis of economically important traits in lentil (Lens culinaris Medik.) germplasm. An International Quarterly Journal of Life 
Sciences, 9(2): 819-822.

Singh, P., Singh, R., Kumar, K. and Singh, D.

K. (2012). Estimates of genetic parameters in diverse genotypes of lentil. Journal of Food Legumes, 25(1): 66-69.

Sirohi, S. P. S., Yadav, R. and Singh, R.(2007). Genetic variability, correlations and path analysis of yield and its component characters in lentil (Lens culinaris L. Medik.). Plant Archives, 7(1): 295-299.

Tadesse, T., Leggesse, T., Mulugeta, B. and Sefera, G. (2014). Correlation and path coefficient analysis of yield and yield components in lentil (Lens culinaris Medik.) germplasm in the highlands of Bale, Ethiopia. International Journal of
Biodiversity and Conservation, 6(1):115-120.

Tyagi, S. D. and Khan, M. H. (2010). Studies on genetic variability and interrelationship among the different traits in Microsperma lentil (Lens culinaris Medik). Journal of Agricultural Biotechnology and Sustainable Development, 2(1): 15-20.

Wright, S. (1921). Correlation and causation. Journal of Agriculture Research, 20 (7): 257-287.

Younis, N., Hanif, M., Sadiq, S., Abbas, G., Asghar, M. J. and Haq, M. A. (2008). Estimates of genetic parameters and path analysis in lentil (Lens culinaris Medik.). Pakistan Journal Agricultural Science, 45(3): 44-48.

\section{How to cite this article:}

Jitendar Kumar Meena, Khajan Singh, P.K.P. Meena, Rajesh Kumar and Deepak Meena. 2020. Studies on Genetic Variability, Correlation and Path Analysis in Lentil (Lens culinaris Medik.) Genotypes. Int.J.Curr.Microbiol.App.Sci. 9(09): 2078-2087.

doi: https://doi.org/10.20546/ijcmas.2020.909.259 